\title{
Avaliação de genótipos de mamoeiro com uso de descritores agronômicos e estimação de parâmetros genéticos
}

\author{
Nágela Lazare Pereira Dias ${ }^{(1)}$, Eder Jorge de Oliveira(2) e Jorge Luiz Loyola Dantas ${ }^{(2)}$
}

\begin{abstract}
(1)Universidade Federal do Recôncavo da Bahia, Campus Universitário de Cruz das Almas, CEP 44380-000 Cruz das Almas, BA. E-mail: nagelap@hotmail.com ${ }^{(2)}$ Embrapa Mandioca e Fruticultura, Rua Embrapa, s/no, Caixa Postal 007, CEP 44380-000 Cruz das Almas, BA. E-mail: eder@cnpmf.embrapa.br, loyola@cnpmf.embrapa.br
\end{abstract}

Resumo - O objetivo deste trabalho foi avaliar descritores quantitativos e estimar parâmetros genéticos em genótipos de mamoeiro (Carica papaya). Foi instalado um experimento em blocos aumentados, para a avaliação de 27 genótipos, entre cultivares, variedades melhoradas e variedades locais, com o uso de 30 descritores relacionados à planta, folhas, flores, frutos e sementes. Após diversos ciclos de melhoramento, os genótipos ainda mostravam ampla variabilidade quanto aos descritores avaliados. A maior parte da variação fenotípica ocorreu em razão da variância genotípica. A herdabilidade variou de 60,48 a $99,05 \%$ e, em $80 \%$ dos casos, foi superior a $80,46 \%$. A razão entre o coeficiente de variação genético e o ambiental foi maior do que a unidade para $63 \%$ das características. Há diferenças agronômicas suficientes para uso dos genótipos "per se" ou como parentais em programas de melhoramento, em razão de agregarem variação genética, qualidade de frutos e tipo agronômico.

Termos para indexação: Carica papaya, desenvolvimento de variedades, melhoramento genético, seleção de parentais, variação genética.

\section{Evaluation of papaya genotypes using agronomic descriptors and estimation of genetic parameters}

\begin{abstract}
The objective of this work was to evaluate quantitative descriptors and to estimate genetic parameters in genotypes of papaya (Carica papaya). An experiment was settled using augmented blocks, for the evaluation of 27 genotypes, among cultivars, improved lines and local varieties with 30 descriptors related to the plant, leaves, flowers, fruit and seeds. After several cycles of breeding, the genotypes still showed broad variability for the evaluated descriptors. Most of the phenotypic variation occurred due to genetic variance. The heritability ranged from 60.48 to $99.05 \%$, and in $80 \%$ cases it was higher than $80.46 \%$. The ratio between genetic and environmental coefficients of variation, was greater than the unity for $63 \%$ traits. There are enough agronomic differences which allow for the use of the genotypes "per se" or as parents in breeding programs because they aggregate genetic variation, fruit quality and agronomic type.
\end{abstract}

Index terms: Carica papaya, variety development, genetic breeding, parental selection, genetic variation.

\section{Introdução}

O mamoeiro pertence ao gênero Carica, que é monotípico e inclui apenas a espécie Carica papaya $\mathrm{L}$. (Badillo, 2000). Atualmente, a produção brasileira de mamão é de 1,8 milhão de toneladas, o que representa $17,64 \%$ do mercado mundial (Food and Agriculture Organization of the United Nations, 2011). Apesar de expressiva, observa-se drástica redução da participação do país no cenário mundial, pois em 1999 ela era de 31,6\%. A área colhida está em torno de 34.379 ha, com destaque para os estados da Bahia, Espírito Santo, Ceará e Rio Grande do Norte, responsáveis por cerca de $92 \%$ da produção nacional (Instituto Brasileiro de Geografia e Estatística, 2011).

As variedades de mamoeiro mais cultivadas pertencem aos grupos Solo e Formosa. Genótipos do grupo Solo apresentam frutos preferidos para exportação, por apresentarem polpa avermelhada e tamanho pequeno, com peso entre 300 e 650 g. Os do grupo Formosa têm polpa laranja-avermelhada e tamanho médio (1.000 a $1.300 \mathrm{~g}$ ), e são formados em sua maioria por híbridos comerciais, mas também podem ser constituídos por linhagens. Contudo, os sistemas de produção são baseados no cultivo de um número reduzido de variedades, o que resulta em restrita variabilidade genética. Esta prática pode levar à maior vulnerabilidade a doenças, pragas e variações edafoclimáticas, e comprometer a sustentabilidade desse cultivo. Assim, a busca pelo aumento da variabilidade genética, por meio do desenvolvimento de novos genótipos, pode garantir maior competitividade e sustentabilidade à cultura do mamoeiro.

O melhoramento genético do mamoeiro no Brasil pode contribuir para aumentar a disponibilidade de

Pesq. agropec. bras., Brasília, v.46, n.11, p.1471-1479, nov. 2011 
variedades com maior produtividade e melhoria na qualidade, e permitir a redução dos custos de produção para garantia de maior competitividade, o que evitaria o caráter itinerante que caracteriza a cultura (Dantas \& Lima, 2001). Como o mamoeiro pode ser autopolinizado sem expressiva perda de vigor (Dantas \& Lima, 2001), a obtenção de linhagens melhoradas, a partir da autofecundação de populações segregantes ou de germoplasma com expressiva variabilidade, é uma técnica viável. Neste sentido, por não apresentar segregação e ter estabilidade fenotípica, estes genótipos podem ser utilizados “per se", nos sistemas de produção ou na produção de híbridos.

O desenvolvimento de variedades de mamoeiro com boas características agronômicas, alta qualidade de frutos e, ainda, com resistência a doenças, é um grande desafio para os melhoristas da cultura, pois essas características não têm sido encontradas em um único genótipo. $\mathrm{O}$ conhecimento da variabilidade genética, disponível no germoplasma da espécie, é um pré-requisito para a indicação de potenciais genitores, para se combinarem alelos relacionados a características de importância econômica, no direcionamento dos cruzamentos em programas de melhoramento.

Alta variação fenotípica para características morfológicas e agronômicas, como tamanho e forma dos frutos, cor, sabor e doçura da polpa, duração do período juvenil, altura da planta, e número de frutos carpeloides, tem sido relatada na literatura (Ocampo et al., 2006; Oliveira et al., 2010). Também têm sido observados baixos níveis de variação genética quanto à resistência a fungos e doenças viróticas (Nishijima, 1994). O conhecimento sobre a natureza e intensidade das variações de origem genética e ambiental é indispensável, para que as ações de melhoramento sejam realizadas de forma eficiente. A melhoria de determinadas características agronômicas depende do conhecimento básico sobre a herança dos caracteres, a variabilidade genética disponível para o melhoramento e as estimativas dos parâmetros genéticos.

Em mamoeiro, as estimativas dos parâmetros genéticos mostram a possibilidade de uso de métodos simples de melhoramento, como a seleção massal quanto à altura da planta, ao diâmetro do caule, ao comprimento do internódio, ao número de folhas e ao índice foliar (Foltran et al., 1993). Em relação à qualidade fisiológica de sementes, há relatos de alta herdabilidade para algumas características (Cardoso et al., 2009). Estimativas de parâmetros genéticos, relacionados a variáveis morfoagronômicas e de qualidade de frutos, também mostram o alto potencial de sucesso com a seleção em populações segregantes, em razão da ampla variabilidade genotípica e dos altos valores de herdabilidade (Silva et al., 2008a). Estudos desta natureza, no entanto, ainda são escassos, apesar de sua importância para subsidiar ações dos programas de melhoramento e do sistema de registro e proteção de cultivares.

O objetivo deste trabalho foi avaliar descritores quantitativos e estimar parâmetros genéticos em genótipos de mamoeiro.

\section{Material e Métodos}

O experimento foi instalado em Cruz das Almas, BA (a $12^{\circ} 48^{\prime} 38^{\prime \prime} \mathrm{S}$ e $39^{\circ} 6^{\prime} 26^{\prime \prime} \mathrm{W}$ ), na área experimental da Embrapa Mandioca e Fruticultura, em julho de 2009, em delineamento de blocos aumentados, com três testemunhas comerciais ('Calimosa', 'Golden' e 'Sunrise'), oito repetições e parcelas formadas por oito plantas, com dados tomados para cada planta da parcela. O plantio foi realizado no espaçamento de 2,0x2,0 m, tendo-se seguido todos os tratos culturais recomendados para a cultura, com uso somente de plantas hermafroditas.

Foram avaliados 27 genótipos de mamoeiro entre cultivares, variedades melhoradas, variedades locais e linhagens pertencentes ao programa de melhoramento genético da Embrapa Mandioca e Fruticultura Tropical (Tabela 1).

As avaliações foram realizadas de outubro de 2009 a dezembro de 2010, com uso de 30 descritores quantitativos, que fazem parte da lista de descritores proposta para o mamoeiro (International Plant Genetic Resources Institute, 1988), que são: altura da planta (m), aos 8 (AP8) e 12 meses (AP12); diâmetro do caule (cm), aos 8 (DC8) e 12 meses (DC12); comprimento médio dos internódios das folhas $(\mathrm{cm})$ no caule, até a altura dos primeiros frutos (DINT); altura de inserção $(\mathrm{cm})$ dos primeiros frutos (APFR); comprimento $(\mathrm{cm})$ das folhas $(\mathrm{CFO})$; largura $(\mathrm{cm})$ das folhas (LFO); razão entre comprimento e largura das folhas (RCFO/LFO); comprimento (cm) do pecíolo da folha (CPEC); comprimento $(\mathrm{cm})$ da corola da flor (CCOR); número de flores por inflorescência (NFL-INFL); comprimento $(\mathrm{cm})$ do pedúnculo da 
inflorescência (CPE-INFL); largura $(\mathrm{cm})$ do fruto (LFR); comprimento ( $\mathrm{cm}$ ) do fruto (CFR); razão entre comprimento e largura de frutos (RCFR/ LFR); massa (g) do fruto (PFR); comprimento (cm) do pedúnculo do fruto (CPE-FR); sólidos solúveis totais, medidos em ${ }^{\circ}$ Brix (SST); acidez total titulável, expressa em gramas de ácido cítrico por $100 \mathrm{~g}$ de suco (ATT); razão entre SST e ATT (Ratio); pH do fruto; comprimento $(\mathrm{cm})$ da cavidade central do fruto (CCAV-FR); firmeza do fruto (FIR-FR), medida em libras, com uso de penetrômetro manual modelo FT 327 (McCormick Fruit Tech, Yakima, WA, EUA); espessura $(\mathrm{cm})$ da casca do fruto (ECAS-FR); massa de matéria fresca ( $\mathrm{g}$ ) das sementes (PFSE); massa de matéria seca (g) das sementes (PSSE); comprimento $(\mathrm{cm})$ de sementes $(\mathrm{CSE})$; largura $(\mathrm{cm})$ de sementes (LSE); e razão entre o comprimento e a largura das sementes (RCSE/LSE).

As medições de características relacionadas a flores e frutos foram realizadas aos 12 meses após o plantio. Foram avaliadas três folhas por planta. $\mathrm{Na}$ análise, foram colhidos, aleatoriamente, oito frutos oriundos de

Tabela 1. Identificação e origem dos 27 genótipos de mamoeiro avaliados.

\begin{tabular}{lcccc}
\hline Genótipo & Tipo & Denominação & Tipo & Procedência \\
\hline CMF008 & Variedade melhorada & DCG593-10 & Formosa & Malásia \\
CMF012 & Variedade melhorada & DCG595-6 & Formosa & Malásia \\
CMF018 & Variedade melhorada & DCG424-6 & Solo & Taiwan \\
CMF020 & Variedade melhorada & DCG424-4 & Formosa & Brasil \\
CMF021 & Cultivar & Solsun & Formosa & Taiwan \\
CMF024 & Cultivar & Conchita & Formosa & Costa Rica \\
CMF041 & Variedade melhorada & JS12 & Formosa & Brasil \\
CMF065 & Variedade melhorada & K77xJSI2 & Solo & Brasil \\
CMF087 & Germoplasma obsoleto & Waimanalo & Solo & Havaí \\
CMF088 & Germoplasma obsoleto & Kapoho purple & Formosa & Havaí \\
CMF092 & Germoplasma obsoleto & Kapoho Green & Solo & Havaí \\
CMF123 & Germoplasma obsoleto & Vermelho Thai & Formosa & Tailândia \\
CMF154 & Germoplasma obsoleto & Maradol & Formosa & Guatemala \\
CMF230 & Variedade local & Ouromel & Solo & Brasil \\
CMF233 & Variedade local & BS Gondo & Solo & Brasil \\
CMF234 & Variedade local & BS SF & Solo & Brasil \\
CMF235 & Variedade local & JTA & Solo & Brasil \\
- & Cultivar & Calimosa & Formosa & Brasil \\
- & Cultivar & Golden & Solo & Brasil \\
- & Cultivar & Sunrise & Solo & Brasil \\
CMF-L12-08 & Linhagem & - & Formosa & Brasil \\
CMF-L62-08 & Linhagem & - & Solo & Brasil \\
CMF-L75-08 & Linhagem & - & Solo & Brasil \\
CMF-L88-08 & Linhagem & - & Formosa & Brasil \\
CMF-L90-08 & Linhagem & - & Formosa & Brasil \\
\hline & & Formosa & Brasil \\
CMFagem & Formosa & Brasil \\
\hline
\end{tabular}

plantas hermafroditas, no estágio 2 de amadurecimento, quando os frutos apresentavam até $25 \%$ da superfície amarela. Entretanto, as análises físico-químicas foram realizadas quando os frutos atingiram o estágio 5, quando os frutos apresentavam $100 \%$ da superfície amarela.

Os dados obtidos foram submetidos à análise de variância, de acordo com o modelo: $Y_{i j}=m+t_{i}+B_{j}+\varepsilon_{j}$, em que: $Y_{\mathrm{ij}}$ é o valor da característica para a i-ésima testemunha, no j-ésimo bloco; $\mathrm{m}$ é a média geral do experimento; $\mathrm{t}_{\mathrm{i}}$ é o efeito do i-ésimo tratamento, que pode ser decomposto em $T_{i}=$ efeito da i-ésima testemunha, com $\mathrm{i}=1,2, \ldots . . \mathrm{t}$ e $\mathrm{G}_{\mathrm{i}}^{\mathrm{j}}=$ efeito do i-ésimo genótipo, com $\mathrm{i}=1,2, \ldots . \mathrm{g}_{\mathrm{j}} ; \mathrm{B}_{\mathrm{j}}$ é o efeito do j-ésimo bloco; $\varepsilon_{\mathrm{ij}}$ é o erro aleatório. Em seguida, foram estimados os parâmetros genéticos: coeficiente de variação experimental $(\mathrm{CVe})$ e genotípico $(\mathrm{CVg})$, relação entre o coeficiente de variação genotípico e experimental $(\mathrm{CVg} / \mathrm{CVe})$, variância fenotípica $\left(\sigma_{\mathrm{f}}^{2}\right)$, genotípica $\left(\sigma_{\mathrm{g}}^{2}\right)$, e herdabilidade dos caracteres avaliados no sentido amplo $\left(\mathrm{h}^{2}\right)$. As médias de genótipos foram comparadas pelo teste de Scott-Knott, a $5 \%$ de probabilidade. Todas as análises estatísticas foram realizadas com auxílio do programa Genes (Cruz, 2006).

\section{Resultados e Discussão}

Com base na análise de variância, observouse diferença significativa em todos os descritores (Tabela 2). O coeficiente de variação experimental (CVe) variou de 3,09 a 50,29\%. De acordo com Gomes (1985), o CVe de descritores relacionados a frutos (PFR e FIR-FR), sementes (PSSE e PFSE) e flores (NFL-INFL) foram classificados como muito altos (>30\%), enquanto o CVe do descritor Ratio foi classificado como alto (22,06\%). Contudo, mesmo com os altos $\mathrm{CVe}$ observados, foi possível constatar diferenças significativas nestes descritores, nos genótipos avaliados.

Além disso, para 80\% dos descritores, os CVe ficaram abaixo de $20 \%$, o que indica boa precisão experimental (Tabela 2). Resultados semelhantes foram observados na análise de características relacionadas à qualidade fisiológica das sementes (Cardoso et al., 2009), bem como em características morfoagronômicas do mamoeiro (Silva et al., 2007, 2008b), em que os autores observaram CVe de baixos a muito altos. 
O coeficiente de variação genética $(\mathrm{CVg})$, calculado como a razão entre o desvio-padrão genético e a média dos genótipos, em percentagem, foi elevado para os descritores CPE-FR, CCAV-FR, NFL-INFL, PSSE, PFR e PFSE. O CVg é um indicador importante da grandeza relativa das mudanças possíveis que podem ser obtidas em cada descritor por meio da seleção, e sua variação tem sido de 12,31 e $60,54 \%$, para as características comprimento do pecíolo das folhas e número de frutos por planta (Oliveira et al., 2010), respectivamente, e de 5,39, para diâmetro do caule aos 260 dias após plantio, e 124,21\% para o número de frutos pentândricos (Silva et al., 2007).

A relação entre $\mathrm{CVg} / \mathrm{CVe}$ foi menor do que a unidade para as características FIR-FR, ECAS-FR, PH, RCFO-LFO, RCSE-LSE, SST, DC8, DC12, LSE, Ratio e CSE. Entretanto, observa-se que as estimativas de herdabilidade no sentido amplo, para

Tabela 2. Resumo da análise de variância dos descritores quantitativos, com as respectivas amplitudes, coeficientes de variação experimental $(\mathrm{CVe})$ e genotípico $(\mathrm{CVg})$, relação entre o coeficiente de variação genotípico e experimental $(\mathrm{CVg} / \mathrm{CVe})$, variância fenotípica $\left(\sigma_{\mathrm{f}}^{2}\right)$, genotípica $\left(\sigma_{\mathrm{g}}^{2}\right)$, e herdabilidade dos caracteres avaliados no sentido amplo $\left(\mathrm{h}^{2}\right)$, em 27 genótipos de mamoeiro.

\begin{tabular}{|c|c|c|c|c|c|c|c|c|c|}
\hline Descritor $^{(1)}$ & QMGen & Mínimo & Máximo & $\sigma_{f}^{2}$ & $\sigma_{g}^{2}$ & $\mathrm{~h}^{2}$ & CVe (\%) & CVg $(\%)$ & $\mathrm{CVg} / \mathrm{CVe}$ \\
\hline AP8 & $1,26 * *$ & 0,65 & 3,80 & 0,1570 & 0,1500 & 95,41 & 11,55 & 18,63 & 1,61 \\
\hline AP12 & $1,60 * *$ & 1,20 & 3,87 & 0,1990 & 0,1910 & 95,55 & 9,97 & 16,34 & 1,64 \\
\hline APFR & $3.709,02 * *$ & 52,00 & 192,00 & 463,6270 & 441,3340 & 95,19 & 10,69 & 16,81 & 1,57 \\
\hline DC8 & $15,23 * *$ & 3,30 & 16,10 & 1,9030 & 1,5310 & 80,46 & 15,83 & 11,36 & 0,72 \\
\hline DC12 & $15,92 * *$ & 5,40 & 18,00 & 1,9900 & 1,6090 & 80,88 & 14,67 & 10,67 & 0,73 \\
\hline DINT & $1,63 * *$ & 1,65 & 4,80 & 0,2030 & 0,1930 & 94,89 & 10,95 & 16,70 & 1,52 \\
\hline $\mathrm{CFO}$ & $230,87 * *$ & 27,00 & 64,50 & 28,8590 & 26,8290 & 92,97 & 9,13 & 11,73 & 1,29 \\
\hline LFO & $562,26 * *$ & 41,00 & 99,00 & 70,2820 & 65,0650 & 92,58 & 9,50 & 11,86 & 1,25 \\
\hline $\mathrm{RCFO} / \mathrm{LFO}$ & $0,01 * *$ & 0,53 & 0,76 & 0,0006 & 0,0005 & 76,82 & 5,30 & 3,41 & 0,64 \\
\hline CPEC & $1.161,81 * *$ & 39,00 & 119,00 & 145,2270 & 135,3180 & 93,18 & 10,48 & 13,69 & 1,31 \\
\hline NFL-INFL & $59,73 * *$ & 1,00 & 18,00 & 7,4660 & 6,7540 & 90,47 & 41,62 & 45,33 & 1,09 \\
\hline CCOR & $343,40 * *$ & 24,40 & 59,20 & 42,9250 & 42,2830 & 98,51 & 6,20 & 17,81 & 2,87 \\
\hline CPE-INFL & $162,09 * *$ & 8,60 & 32,60 & 20,2610 & 20,0680 & 99,05 & 6,73 & 24,24 & 3,60 \\
\hline CPE-FR & $20,02 * *$ & 2,43 & 11,07 & 2,5020 & 2,3790 & 95,06 & 17,73 & 27,50 & 1,55 \\
\hline PFR & $1,78 * *$ & 0,15 & 2,45 & 0,2220 & 0,2130 & 95,94 & 31,16 & 53,58 & 1,72 \\
\hline CFR & $93,43 * *$ & 9,20 & 28,70 & 11,6780 & 11,1660 & 95,61 & 11,72 & 19,34 & 1,65 \\
\hline LFR & $40,42 * *$ & 5,50 & 17,30 & 5,0530 & 4,8900 & 96,77 & 11,40 & 22,08 & 1,94 \\
\hline RCFR/LFR & $0,53 * *$ & 1,10 & 2,76 & 0,0663 & 0,0600 & 90,50 & 12,76 & 13,92 & 1,09 \\
\hline ECAS-FR & $0,0007 * *$ & 0,07 & 0,22 & 0,0001 & 0,0001 & 66,15 & 13,52 & 6,68 & 0,49 \\
\hline CCAV-FR & $26,19 * *$ & 2,74 & 11,55 & 3,2740 & 3,1740 & 96,95 & 15,67 & 31,21 & 1,99 \\
\hline FIR-FR & $1,33 * *$ & 0,20 & 4,40 & 0,1660 & 0,1000 & 60,48 & 50,29 & 22,00 & 0,44 \\
\hline ATT & $0,0021 * *$ & 0,04 & 0,18 & 0,0003 & 0,0002 & 89,76 & 19,39 & 20,29 & 1,05 \\
\hline SST & $9,87 * *$ & 5,00 & 13,00 & 1,2340 & 0,9860 & 79,91 & 14,55 & 10,26 & 0,71 \\
\hline Ratio & $7.106,60 * *$ & 40,10 & 278,40 & 888,3250 & 772,7590 & 86,99 & 22,06 & 20,17 & 0,91 \\
\hline $\mathrm{pH}$ & $0,11 * *$ & 4,68 & 5,92 & 0,0140 & 0,0110 & 74,91 & 3,09 & 1,89 & 0,61 \\
\hline PFSE & $5.524,06^{* *}$ & 6,48 & 173,00 & 690,5080 & 654,7360 & 94,82 & 38,61 & 58,40 & 1,51 \\
\hline PSSE & $183,12 * *$ & 2,71 & 32,29 & 22,8900 & 21,9250 & 95,79 & 30,50 & 51,42 & 1,69 \\
\hline CSE & $0,0112 * *$ & 0,47 & 0,71 & 0,0014 & 0,0012 & 88,22 & 6,32 & 6,12 & 0,97 \\
\hline LSE & $0,0101 * *$ & 0,32 & 0,59 & 0,0013 & 0,0011 & 86,24 & 9,04 & 8,00 & 0,89 \\
\hline RCSE/LSE & $0,0377 * *$ & 1,15 & 1,72 & 0,0047 & 0,0037 & 79,47 & 6,28 & 4,37 & 0,70 \\
\hline
\end{tabular}

${ }^{(1)}$ AP8 e AP12, altura da planta (m) aos 8 e 12 meses, respectivamente; APFR, altura (cm) dos primeiros frutos; DC8 e DC12, diâmetro do caule (cm) aos 8 e 12 meses, respectivamente; DINT, comprimento médio dos internódios das folhas (cm) no caule, até a altura dos primeiros frutos; CFO, comprimento das folhas (cm); LFO, largura das folhas (cm); RCFO/LFO, razão entre comprimento e largura das folhas; CPEC, comprimento do pecíolo da folha (cm); NFL-INFL, número de flores por inflorescência; CCOR, comprimento da corola da flor (cm); CPE-INFL, comprimento do pedúnculo da inflorescência (cm); CPE-FR, comprimento do pedúnculo do fruto (cm); PFR, massa do fruto (g); CFR, comprimento do fruto (cm); LFR, largura do fruto (cm); RCFR/LFR, razão entre comprimento e largura de fruto; ECAS-FR, espessura da casca do fruto (cm); CCAV-FR, comprimento da cavidade central do fruto (cm); FIR-FR, firmeza do fruto (libras); ATT, acidez total titulável (g de ácido cítrico por 100 g); SST, sólidos solúveis totais ( ${ }^{\circ}$ Brix); Ratio, razão entre SST e ATT; pH do fruto; PFSE, massa de matéria fresca das sementes (g); PSSE, massa de matéria seca das sementes (g); CSE, comprimento de sementes (cm); LSE, largura de sementes (cm); RCSE/LSE, razão entre comprimento e largura de sementes. QMGen, quadrado médio de genótipos. **Significativo a 1\% de probabilidade, pelo teste $\mathrm{F}$. 
estas características, foram de média à alta magnitude, com variação de $60,48 \%$ (FIR-FR) a 88,22\% (CSE) (Tabela 2). Isto indica que, embora o processo seletivo favoreça em magnitude as demais características, em longo prazo é possível obter ganhos tão ou mais expressivos para FIR-FR, ECAS-FR, PH, RCFO-LFO, RCSE-LSE, SST, DC8, DC12, LSE, Ratio e CSE, em razão da semelhança entre os $\mathrm{CVg}$ com as outras características.

No entanto, para $63 \%$ das características avaliadas, a relação $\mathrm{CVg} / \mathrm{CVe}$ foi maior do que a unidade, com destaque para APFR, AP8 e AP12, que são características que influenciam diretamente a colheita, já que plantas de menor estatura facilitam este processo. As características CFR, PFR, LFR e CCAV-FR também são importantes por influenciar o padrão de frutos. No caso do mamão do tipo Solo, a experiência de produtores da região indica que frutos com calibre 15/18 são mais valorizados no mercado e, portanto, merecem o foco da pesquisa relacionada ao desenvolvimento de novas cultivares.

Os altos valores de herdabilidade indicam que, havendo interesse dos programas de melhoramento, há possibilidade de ganhos expressivos no processo de seleção quanto à maioria dos descritores (Tabela 2). Estes resultados são consistentes com as estimativas de herdabilidade no sentido restrito, em populações segregantes (Singh \& Kumar, 2010), cujos valores variaram de $50,44 \%$, quanto ao número de frutos por planta, e $96 \%$, quanto ao peso de frutos, o que é indício de alta viabilidade para o emprego de métodos simples de seleção em populações segregantes. Entretanto, na Venezuela e em Guadalupe, Granada e Trinidad e Tobago, no Caribe, a seleção realizada em germoplasma local indicou alta herdabilidade quanto à resistência a doenças, porém a alta heterogeneidade destes materiais não permitiu produção uniforme e com boas características organolépticas de frutos, tendo sido necessário o desenvolvimento de um programa de melhoramento genético que combinasse resistência à bacteriose com boa qualidade de frutos (Ocampo et al., 2006).

Silva et al. (2008b) também obtiveram estimativas de herdabilidade acima de $80 \%$ para diversas características morfoagronômicas do mamoeiro como altura de plantas, diâmetro de caule, altura de inserção dos primeiros frutos, número de flores (totais, carpeloides, pentândricas), número de frutos (normais, carpeloides, pentândricos), massa, comprimento e diâmetro de frutos, produtividade, e firmeza interna e externa dos frutos.

Entre os componentes de variância, observou-se maior participação da variância genotípica em todas as características estudadas (Tabela 2). Segundo Cruz \& Carneiro (2003), a utilização de parâmetros genéticos no melhoramento de plantas permite a identificação da variabilidade genética e análise da eficiência de estratégias de melhoramento utilizadas para maximizar os ganhos e, ao mesmo tempo, permite manter a base genética da população.

Os genótipos Sunrise e CMF018 apresentaram a maior altura de planta (acima de 3,32 m) (Tabela 3). Os genótipos CMF008, CMF154, CMF020, L90-08, CMF041 e L75-08 apresentaram altura de planta inferior a 2,30 m. De acordo com Nakasone \& Lamoureux (1982), plantas muito altas são indesejáveis por apresentar internódios muito longos e, com isso, maior espaço entre frutos, menor produtividade e longevidade de produção. Contudo, ao se analisar a altura dos primeiros frutos, observouse que genótipos de porte alto (CMF018), baixo (CMF008, CMF154 e CMF020) e mediano (CMF088 e CMF123) apresentaram frutificação próxima à da observada no tipo Solo.

Com relação ao diâmetro do caule, verificou-se ampla variação entre os genótipos com maior diâmetro, acima de 12,99 cm (CMF123, CMF234, L88-08, L90-08, CMF024, CMF065, Sunrise e CMF018), e os com menor diâmetro (CMF008 e CMF041), com variação de 7,99 a $9,03 \mathrm{~cm}$. Fraife Filho et al. (2001) e Silva et al. (2007) indicam que a seleção de plantas com maior diâmetro do caule pode resultar em plantas mais produtivas, em virtude da alta correlação genética entre essas características. Portanto, este descritor deve ser utilizado para compor índices de seleção em programas de melhoramento.

Onze genótipos estiveram entre os de menor DINT: CMF087, CMF235, CMF123, CMF018, CMF008, CMF234, CMF020, L48-08, CMF088, L30-08 e Calimosa (Tabela 3). Esta característica está associada ao maior número de flores por planta e, portanto, maior número de frutos. Maiores comprimentos de folhas foram acompanhados por maior largura, sobretudo nos genótipos CMF020, CMF021, CMF154, L62-08, Calimosa, CMF123 e L12-08. Exceção a esta tendência 
foi observada na linhagem L30-08 e nas variedades melhoradas CMF041 e CMF065.

Os genótipos contrastantes quanto ao comprimento do pecíolo foram Calimosa, L62-08, CMF021, L88-08, L48-08 e L12-08, que apresentaram valores acima de 96,86 cm, e CMF008, CMF041, CMF018 e CMF088, com valores entre 62,89 e 68,00 cm (Tabela 3). Em relação ao NFL-INFL, observou-se que os genótipos CMF008 e L12-08 apresentaram mais de 11 flores por inflorescência, em comparação aos genótipos CMF012, CMF024, CMF154, CMF021 e CMF088, que apresentaram menos de três. Ampla diversidade também foi observada quanto ao comprimento da corola $(28,33$ a $53,75 \mathrm{~mm})$ e ao comprimento do pedúnculo da inflorescência $(10,65$ a $30,64 \mathrm{~mm}$ ). Não se observou relação entre estas duas últimas características e a classificação dos genótipos nos grupos Solo e Formosa.

$\mathrm{O}$ comprimento do pedúnculo dos frutos variou de 3,75 (CMF087) a 10,71 cm (CMF024) (Tabela 4). Com relação à massa dos frutos, observou-se a formação de quatro grupos, e os genótipos CMF123, CMF024 e CMF012 foram agrupados com médias entre 1,82 e 1,89 kg, e CMF008, CMF154 e CMF021 com médias de 1,31 a 1,41 kg. Nestes dois agrupamentos, todos os genótipos pertencem ao tipo Formosa. No terceiro agrupamento de médias, observaram-se tanto genótipos do tipo Solo (L30-08, CMF233 e L48-08), quanto do grupo Formosa (L62-08, L88-08, CMF041, Calimosa, CMF088, L75-08, CMF020, L12-08 e L90-08), com massas entre 0,69 e 1,00 kg. Os genótipos CMF230, Golden, CMF234, CMF087, Sunrise, CMF018, CMF092, CMF235 e CMF065, classificados como

Tabela 3. Média dos descritores ${ }^{(1)}$ de mamoeiro relacionados à planta, folhas, flores e inflorescências, avaliados em 27 genótipos de mamoeiro.

\begin{tabular}{|c|c|c|c|c|c|c|c|c|c|c|c|c|c|}
\hline Genótipo & AP8 & AP12 & APFR & DC8 & DC12 & DINT & $\mathrm{CFO}$ & LFO & RCFO/LFO & CPEC & NFL-INFL & CCOR & CPE-INFL \\
\hline Calimosa & $1,88 \mathrm{~d}$ & $2,45 \mathrm{~d}$ & $130,86 \mathrm{c}$ & $10,69 b$ & $12,36 b$ & $2,43 d$ & $50,10 \mathrm{a}$ & $78,29 a$ & $0,64 b$ & $96,86 a$ & $7,86 \mathrm{~b}$ & $38,14 \mathrm{f}$ & $25,54 \mathrm{~b}$ \\
\hline CMF008 & $1,24 \mathrm{~g}$ & $1,63 \mathrm{~g}$ & $60,67 \mathrm{e}$ & $6,23 \mathrm{c}$ & 7,99d & $2,17 \mathrm{~d}$ & $36,85 \mathrm{~d}$ & $54,12 d$ & $0,68 \mathrm{a}$ & $62,89 \mathrm{e}$ & $11,58 \mathrm{a}$ & $48,07 \mathrm{c}$ & $24,91 b$ \\
\hline CMF012 & $2,48 \mathrm{c}$ & $3,11 \mathrm{~b}$ & $162,50 \mathrm{a}$ & $10,85 b$ & $12,29 b$ & $2,68 \mathrm{c}$ & $46,08 b$ & $68,17 \mathrm{c}$ & $0,68 \mathrm{a}$ & $74,50 \mathrm{~d}$ & $1,00 \mathrm{~d}$ & $53,75 \mathrm{a}$ & $18,04 \mathrm{f}$ \\
\hline CMF018 & $2,61 b$ & $3,32 \mathrm{a}$ & $96,50 \mathrm{~d}$ & $12,90 \mathrm{a}$ & $13,98 \mathrm{a}$ & $2,17 \mathrm{~d}$ & $41,08 \mathrm{c}$ & $61,93 \mathrm{c}$ & $0,67 \mathrm{a}$ & $64,50 \mathrm{e}$ & $5,67 \mathrm{c}$ & $36,53 \mathrm{~g}$ & $21,70 \mathrm{c}$ \\
\hline CMF020 & $1,78 \mathrm{e}$ & $2,15 \mathrm{e}$ & $104,50 \mathrm{~d}$ & $10,08 \mathrm{~b}$ & $10,70 \mathrm{c}$ & $2,25 \mathrm{~d}$ & $50,28 \mathrm{a}$ & $75,76 a$ & $0,67 \mathrm{a}$ & $93,88 \mathrm{~b}$ & $6,13 \mathrm{c}$ & $38,30 \mathrm{f}$ & $20,23 d$ \\
\hline CMF021 & $2,01 \mathrm{~d}$ & $3,00 \mathrm{~b}$ & $121,54 \mathrm{c}$ & $10,10 \mathrm{~b}$ & $11,38 b$ & $3,89 \mathrm{a}$ & $48,52 \mathrm{a}$ & $77,06 a$ & $0,64 b$ & $97,92 \mathrm{a}$ & $2,00 \mathrm{~d}$ & $44,67 d$ & $19,00 \mathrm{e}$ \\
\hline CMF024 & $3,05 \mathrm{a}$ & $3,24 b$ & $160,69 a$ & $13,19 \mathrm{a}$ & $13,39 \mathrm{a}$ & $2,69 \mathrm{c}$ & $46,29 b$ & $68,23 \mathrm{c}$ & $0,69 \mathrm{a}$ & $83,77 \mathrm{c}$ & $1,00 \mathrm{~d}$ & $51,28 \mathrm{~b}$ & $18,64 \mathrm{e}$ \\
\hline CMF041 & $1,90 \mathrm{~d}$ & $2,21 \mathrm{e}$ & $121,00 \mathrm{c}$ & $10,20 \mathrm{~b}$ & $9,03 \mathrm{~d}$ & $2,74 \mathrm{c}$ & $32,83 \mathrm{~d}$ & $54,00 \mathrm{~d}$ & $0,61 \mathrm{c}$ & $63,67 \mathrm{e}$ & $4,67 \mathrm{c}$ & $28,33 \mathrm{i}$ & $25,53 b$ \\
\hline CMF065 & $2,08 \mathrm{~d}$ & $3,05 \mathrm{~b}$ & $128,86 \mathrm{c}$ & $12,29 \mathrm{a}$ & $13,66 \mathrm{a}$ & $2,88 \mathrm{c}$ & $42,69 \mathrm{c}$ & $70,21 b$ & $0,61 \mathrm{c}$ & $89,57 b$ & $5,43 \mathrm{c}$ & $34,71 \mathrm{~g}$ & $20,40 \mathrm{~d}$ \\
\hline CMF087 & $2,27 \mathrm{c}$ & $3,11 \mathrm{~b}$ & $141,57 b$ & $11,60 \mathrm{a}$ & $12,37 b$ & $1,93 d$ & $34,57 d$ & $54,29 d$ & $0,64 b$ & $73,57 d$ & $5,71 \mathrm{c}$ & $37,37 f$ & $17,37 f$ \\
\hline CMF088 & $2,06 \mathrm{~d}$ & $2,66 \mathrm{c}$ & $97,00 d$ & $9,80 \mathrm{~b}$ & $11,36 b$ & $2,30 \mathrm{~d}$ & $39,48 \mathrm{c}$ & $60,78 \mathrm{c}$ & $0,65 \mathrm{a}$ & $68,00 \mathrm{e}$ & $3,38 \mathrm{~d}$ & $39,93 \mathrm{e}$ & $19,50 \mathrm{e}$ \\
\hline CMF092 & $2,44 \mathrm{c}$ & $3,22 b$ & $144,25 b$ & $11,34 \mathrm{a}$ & $12,51 b$ & $2,64 \mathrm{c}$ & $37,58 \mathrm{~d}$ & $57,06 \mathrm{~d}$ & $0,66 \mathrm{a}$ & $71,25 \mathrm{~d}$ & $5,50 \mathrm{c}$ & $41,08 \mathrm{e}$ & $18,58 \mathrm{e}$ \\
\hline CMF123 & $2,24 \mathrm{c}$ & $2,87 \mathrm{c}$ & $105,38 d$ & $11,60 \mathrm{a}$ & $12,86 \mathrm{a}$ & $2,15 d$ & $52,23 \mathrm{a}$ & $79,93 a$ & $0,65 \mathrm{a}$ & $91,63 b$ & $7,13 b$ & $34,88 \mathrm{~g}$ & $20,58 d$ \\
\hline CMF154 & $1,51 \mathrm{f}$ & $2,04 \mathrm{f}$ & $101,17 d$ & $9,56 \mathrm{~b}$ & $10,47 \mathrm{c}$ & $2,76 \mathrm{c}$ & $49,97 \mathrm{a}$ & $77,20 \mathrm{a}$ & $0,65 b$ & $92,83 \mathrm{~b}$ & $1,00 \mathrm{~d}$ & $31,76 \mathrm{~h}$ & $11,40 \mathrm{k}$ \\
\hline CMF230 & $1,79 \mathrm{e}$ & $2,46 \mathrm{~d}$ & $125,38 \mathrm{c}$ & $10,13 b$ & $11,11 \mathrm{~b}$ & $2,73 \mathrm{c}$ & $42,80 \mathrm{c}$ & $63,99 \mathrm{c}$ & $0,67 \mathrm{a}$ & $81,75 \mathrm{c}$ & $4,50 \mathrm{c}$ & $31,38 \mathrm{~h}$ & $10,65 \mathrm{k}$ \\
\hline CMF233 & $2,41 \mathrm{c}$ & $2,77 \mathrm{c}$ & $131,25 \mathrm{c}$ & $10,84 b$ & $11,76 \mathrm{~b}$ & $2,51 \mathrm{c}$ & $44,35 b$ & $62,48 \mathrm{c}$ & $0,71 \mathrm{a}$ & $81,25 \mathrm{c}$ & $6,00 \mathrm{c}$ & $35,20 \mathrm{~g}$ & $15,43 \mathrm{~h}$ \\
\hline CMF234 & $2,42 \mathrm{c}$ & $2,81 \mathrm{c}$ & $133,75 \mathrm{c}$ & $12,25 \mathrm{a}$ & $12,94 \mathrm{a}$ & $2,24 d$ & $44,64 b$ & $66,56 \mathrm{c}$ & $0,67 \mathrm{a}$ & $90,25 b$ & $5,38 \mathrm{c}$ & $31,30 \mathrm{~h}$ & $16,30 \mathrm{~g}$ \\
\hline CMF235 & $2,01 d$ & $2,43 d$ & $129,20 \mathrm{c}$ & $11,12 \mathrm{a}$ & $11,70 \mathrm{~b}$ & $2,13 \mathrm{~d}$ & $39,70 \mathrm{c}$ & $62,48 \mathrm{c}$ & $0,64 b$ & $83,60 \mathrm{c}$ & $5,00 \mathrm{c}$ & $31,36 \mathrm{~h}$ & $17,56 \mathrm{f}$ \\
\hline Golden & $2,36 \mathrm{c}$ & $2,71 \mathrm{c}$ & $141,00 \mathrm{~b}$ & $11,21 \mathrm{a}$ & $11,84 b$ & $2,75 \mathrm{c}$ & $40,46 c$ & $62,77 \mathrm{c}$ & $0,64 b$ & $81,43 \mathrm{c}$ & $7,29 b$ & $34,17 \mathrm{~g}$ & $17,46 f$ \\
\hline CMF-L12-08 & $1,79 \mathrm{e}$ & $2,43 d$ & $125,83 \mathrm{c}$ & $10,25 b$ & $12,08 b$ & $3,12 b$ & $54,09 \mathrm{a}$ & $83,05 \mathrm{a}$ & $0,65 \mathrm{a}$ & $102,48 \mathrm{a}$ & $12,75 \mathrm{a}$ & $34,52 \mathrm{~g}$ & $30,64 a$ \\
\hline CMF-L30-08 & $1,91 \mathrm{~d}$ & $2,45 \mathrm{~d}$ & $122,13 \mathrm{c}$ & $10,63 b$ & $11,46 b$ & $2,41 d$ & $43,96 b$ & $75,34 \mathrm{a}$ & $0,59 \mathrm{c}$ & $92,50 \mathrm{~b}$ & $5,88 \mathrm{c}$ & $34,03 \mathrm{~g}$ & $16,90 \mathrm{~g}$ \\
\hline CMF-L48-08 & $1,71 \mathrm{e}$ & $2,39 d$ & $120,25 \mathrm{c}$ & $10,31 b$ & $12,38 b$ & $2,27 d$ & $45,23 b$ & $72,31 b$ & $0,63 b$ & $102,38 \mathrm{a}$ & $6,63 b$ & $37,53 \mathrm{f}$ & $16,45 \mathrm{~g}$ \\
\hline CMF-L62-08 & $1,96 \mathrm{~d}$ & $2,76 \mathrm{c}$ & $138,43 b$ & $10,79 b$ & $11,54 b$ & $3,21 b$ & $50,20 \mathrm{a}$ & $77,93 \mathrm{a}$ & $0,64 b$ & $97,86 a$ & $5,71 \mathrm{c}$ & $31,40 \mathrm{~h}$ & $19,37 \mathrm{e}$ \\
\hline CMF-L 75-08 & $1,68 \mathrm{e}$ & $2,30 \mathrm{e}$ & $119,38 \mathrm{c}$ & $9,69 b$ & $9,94 \mathrm{c}$ & $2,76 \mathrm{c}$ & $43,70 b$ & $66,38 \mathrm{c}$ & $0,66 \mathrm{a}$ & $88,14 b$ & $5,52 \mathrm{c}$ & $28,78 \mathrm{i}$ & $12,70 \mathrm{j}$ \\
\hline CMF-L88-08 & $2,14 \mathrm{~d}$ & $2,82 \mathrm{c}$ & $147,14 b$ & $11,69 \mathrm{a}$ & $12,99 \mathrm{a}$ & $3,27 b$ & $47,06 b$ & $71,26 b$ & $0,66 \mathrm{a}$ & $100,43 a$ & $7,57 \mathrm{~b}$ & $30,57 \mathrm{~h}$ & $13,94 \mathrm{i}$ \\
\hline CMF-L90-08 & $1,73 \mathrm{e}$ & $2,20 \mathrm{e}$ & $119,13 \mathrm{c}$ & $11,95 \mathrm{a}$ & $13,10 \mathrm{a}$ & $3,38 b$ & $46,98 b$ & $72,23 b$ & $0,65 \mathrm{a}$ & $87,25 b$ & $8,38 \mathrm{~b}$ & $29,73 \mathrm{i}$ & $14,03 \mathrm{i}$ \\
\hline Sunrise & $2,68 \mathrm{~b}$ & $3,53 \mathrm{a}$ & $144,67 b$ & $12,88 \mathrm{a}$ & $13,87 \mathrm{a}$ & $2,56 \mathrm{c}$ & $40,63 \mathrm{c}$ & $62,82 \mathrm{c}$ & $0,65 b$ & $79,50 \mathrm{c}$ & $6,17 \mathrm{c}$ & $37,20 \mathrm{f}$ & $16,20 \mathrm{~g}$ \\
\hline
\end{tabular}

(1)AP8 e AP12, altura da planta (m) aos 8 e 12 meses, respectivamente; APFR, altura (cm) dos primeiros frutos; DC8 e DC12, diâmetro do caule (cm) aos 8 e 12 meses, respectivamente; DINT, comprimento médio dos internódios das folhas (cm) no caule, até a altura dos primeiros frutos; CFO, comprimento das folhas (cm); LFO, largura das folhas (cm); RCFO/LFO, razão entre comprimento e largura das folhas; CPEC, comprimento do pecíolo da folha (cm); NFL-INFL, número de flores por inflorescência; CCOR, comprimento da corola da flor (cm); CPE-INFL, comprimento do pedúnculo da inflorescência (cm). Médias seguidas de letras iguais não diferem entre si pelo teste Scott-Knott, a 5\% de probabilidade. 
tipo Solo, apresentaram massas com variação de 0,26 a $0,55 \mathrm{~kg}$.

A massa de frutos indicou que existe potencial para a seleção de plantas quanto à produção de frutos com padrão que atenda tanto o mercado nacional, que exige frutos com massa entre 0,80 e $1,50 \mathrm{~kg}$, quanto o mercado externo, que exige massa em torno de $0,50 \mathrm{~kg}$.

Ao analisar acessos de germoplasma oriundos da Venezuela, Ocampo et al. (2006) também observaram alto polimorfismo para esta característica, com massas entre 0,12 e $3,74 \mathrm{~kg}$. No entanto, Dantas \& Lima (2001) observaram massa média de frutos de 0,28 a $0,85 \mathrm{~kg}$, em acessos do grupo Solo, e 0,71 a 2,2 kg no grupo Formosa. Os autores ressaltaram que a alta heterogeneidade do material avaliado mostra alto potencial para seleção quanto ao aumento da massa dos frutos, apesar da marcante influência do ambiente na expressão do caráter. A alta herdabilidade observada no presente trabalho (acima de 95\%, Tabela 2) corrobora a possibilidade de sucesso na seleção.

As dimensões dos frutos também foram bastante variáveis, tanto para comprimento - de $11,70 \mathrm{~cm}$ (CMF230) a 24,14 cm (CMF123) -, quanto para largura de frutos - de 6,91 cm (CMF230) a 15,89 cm (CMF012). A classificação do mamoeiro nos tipos Solo e Formosa é bastante subjetiva, quando se trata de genótipos com alta variabilidade quanto ao tamanho e à forma dos frutos, como os avaliados no presente trabalho. Apesar disso, os resultados do agrupamento de médias indicam consistência no uso dos descritores PFR, CFR e LFR para a classificação dos genótipos de mamoeiro em tipo Solo ou Formosa.

A espessura da casca dos frutos apresentou menor amplitude de valores (Tabela 4), com formação de apenas dois agrupamentos: os genótipos CMF230, CMF123 e Golden apresentaram os maiores valores

Tabela 4. Média dos descritores ${ }^{(1)}$ de mamoeiro, relacionados aos frutos avaliados em 27 genótipos de mamoeiro.

\begin{tabular}{|c|c|c|c|c|c|c|c|c|c|c|c|c|}
\hline Genótipo & CPE-FR & PFR & CFR & LFR & RCFR/LFR & ECAS-FR & CCAV-FR & FIR-FR & ATT & SST & Ratio & $\mathrm{pH}$ \\
\hline Calimosa & $6,04 \mathrm{c}$ & $0,85 \mathrm{c}$ & $19,48 \mathrm{~d}$ & $9,59 \mathrm{f}$ & $2,05 \mathrm{a}$ & $0,11 \mathrm{~b}$ & $4,96 \mathrm{f}$ & $0,64 \mathrm{~b}$ & $0,08 \mathrm{~b}$ & $9,48 b$ & $124,33 b$ & $5,56 \mathrm{a}$ \\
\hline CMF008 & $4,91 d$ & $1,31 \mathrm{~b}$ & $19,70 \mathrm{c}$ & $11,87 \mathrm{~d}$ & $1,70 \mathrm{c}$ & $0,10 \mathrm{~b}$ & $6,89 \mathrm{~d}$ & $1,80 \mathrm{a}$ & $0,14 \mathrm{a}$ & $8,59 \mathrm{c}$ & $62,46 \mathrm{~d}$ & $5,44 \mathrm{a}$ \\
\hline CMF012 & $7,95 b$ & $1,99 a$ & $20,04 c$ & $15,89 \mathrm{a}$ & $1,26 \mathrm{~d}$ & $0,11 \mathrm{~b}$ & $10,64 \mathrm{a}$ & $1,50 \mathrm{~b}$ & $0,07 \mathrm{c}$ & $9,38 b$ & $144,86 a$ & $5,57 \mathrm{a}$ \\
\hline CMF018 & $8,35 b$ & $0,45 \mathrm{~d}$ & $14,40 \mathrm{e}$ & $8,06 \mathrm{~g}$ & $1,80 \mathrm{~b}$ & $0,11 \mathrm{~b}$ & $3,87 \mathrm{~g}$ & $1,43 b$ & $0,07 \mathrm{c}$ & $9,65 b$ & $152,64 \mathrm{a}$ & $5,24 \mathrm{~b}$ \\
\hline CMF020 & $6,33 \mathrm{c}$ & $0,95 \mathrm{c}$ & $19,01 \mathrm{c}$ & $10,25 \mathrm{e}$ & $1,86 \mathrm{~b}$ & $0,11 \mathrm{~b}$ & $5,43 \mathrm{f}$ & $1,85 \mathrm{a}$ & $0,09 b$ & $8,15 c$ & $96,66 c$ & $5,40 \mathrm{~b}$ \\
\hline CMF021 & $5,25 \mathrm{~d}$ & $1,41 \mathrm{~b}$ & $17,18 \mathrm{~d}$ & $14,05 \mathrm{~b}$ & $1,83 \mathrm{~b}$ & $0,11 \mathrm{~b}$ & $5,39 \mathrm{f}$ & $1,13 b$ & $0,09 b$ & $10,03 b$ & $132,23 b$ & $5,56 \mathrm{a}$ \\
\hline CMF024 & $10,71 \mathrm{a}$ & $1,82 \mathrm{a}$ & $21,96 \mathrm{~b}$ & $14,38 \mathrm{~b}$ & $1,52 \mathrm{c}$ & $0,10 \mathrm{~b}$ & $9,57 \mathrm{~b}$ & $2,01 \mathrm{a}$ & $0,08 \mathrm{~b}$ & $7,35 \mathrm{c}$ & $100,68 \mathrm{c}$ & $5,60 \mathrm{a}$ \\
\hline CMF041 & $4,86 \mathrm{~d}$ & $0,82 \mathrm{c}$ & $18,24 \mathrm{e}$ & $9,33 \mathrm{f}$ & $1,76 \mathrm{c}$ & $0,12 b$ & $5,42 \mathrm{f}$ & $1,75 \mathrm{a}$ & $0,07 \mathrm{c}$ & $10,38 \mathrm{a}$ & $151,79 a$ & $5,37 \mathrm{~b}$ \\
\hline CMF065 & $6,91 \mathrm{c}$ & $0,55 \mathrm{~d}$ & $14,40 \mathrm{e}$ & $8,71 \mathrm{f}$ & $1,65 c$ & $0,11 \mathrm{~b}$ & $4,98 \mathrm{f}$ & $1,04 b$ & $0,07 \mathrm{c}$ & $11,53 \mathrm{a}$ & $185,89 \mathrm{a}$ & $5,64 \mathrm{a}$ \\
\hline CMF087 & $3,75 \mathrm{e}$ & $0,41 \mathrm{~d}$ & $12,21 \mathrm{f}$ & $8,11 \mathrm{~g}$ & $1,51 \mathrm{c}$ & $0,11 \mathrm{~b}$ & $4,71 \mathrm{~g}$ & $1,45 b$ & $0,06 \mathrm{c}$ & $10,58 \mathrm{a}$ & $168,84 \mathrm{a}$ & $5,48 \mathrm{a}$ \\
\hline CMF088 & $6,39 c$ & $0,87 \mathrm{c}$ & $18,98 \mathrm{c}$ & $10,13 \mathrm{e}$ & $1,90 \mathrm{~b}$ & $0,12 \mathrm{~b}$ & $5,94 \mathrm{e}$ & $1,36 \mathrm{~b}$ & $0,07 \mathrm{c}$ & $10,35 \mathrm{a}$ & $158,69 \mathrm{a}$ & $5,41 \mathrm{~b}$ \\
\hline CMF092 & $5,19 \mathrm{~d}$ & $0,50 \mathrm{~d}$ & $14,08 \mathrm{e}$ & $8,89 \mathrm{f}$ & $1,59 \mathrm{c}$ & $0,10 \mathrm{~b}$ & $5,09 \mathrm{f}$ & $1,25 \mathrm{~b}$ & $0,07 \mathrm{c}$ & $11,25 \mathrm{a}$ & $171,80 \mathrm{a}$ & $5,26 \mathrm{~b}$ \\
\hline CMF123 & $6,67 \mathrm{c}$ & $1,82 \mathrm{a}$ & $24,14 \mathrm{a}$ & $12,84 \mathrm{c}$ & $1,88 \mathrm{~b}$ & $0,13 \mathrm{a}$ & $7,13 d$ & $1,36 \mathrm{~b}$ & $0,08 b$ & $9,45 b$ & $134,05 \mathrm{~b}$ & $5,34 \mathrm{~b}$ \\
\hline CMF154 & $4,79 d$ & $1,33 b$ & $20,05 \mathrm{c}$ & $13,23 \mathrm{c}$ & $1,52 \mathrm{c}$ & $0,11 \mathrm{~b}$ & $8,41 \mathrm{c}$ & $1,99 \mathrm{a}$ & $0,09 b$ & $9,08 \mathrm{c}$ & $102,28 \mathrm{c}$ & $5,31 \mathrm{~b}$ \\
\hline CMF230 & $3,79 \mathrm{e}$ & $0,26 \mathrm{~d}$ & $11,70 \mathrm{f}$ & $6,91 \mathrm{~g}$ & $1,70 \mathrm{c}$ & $0,13 \mathrm{a}$ & $3,69 \mathrm{~g}$ & $1,10 \mathrm{~b}$ & $0,07 \mathrm{c}$ & $9,73 b$ & $145,18 \mathrm{a}$ & $5,62 \mathrm{a}$ \\
\hline CMF233 & $4,34 \mathrm{e}$ & $0,76 \mathrm{c}$ & $17,34 \mathrm{~d}$ & $9,88 \mathrm{e}$ & $1,23 \mathrm{~d}$ & $0,12 b$ & $9,51 \mathrm{~b}$ & $1,01 \mathrm{~b}$ & $0,08 \mathrm{~b}$ & $9,63 b$ & $121,23 b$ & $5,50 \mathrm{a}$ \\
\hline CMF234 & $4,43 \mathrm{e}$ & $0,40 \mathrm{~d}$ & $13,00 \mathrm{f}$ & $7,73 \mathrm{~g}$ & $1,73 \mathrm{c}$ & $0,11 \mathrm{~b}$ & $4,49 \mathrm{~g}$ & $2,00 \mathrm{a}$ & $0,06 \mathrm{c}$ & $10,48 \mathrm{a}$ & $164,15 \mathrm{a}$ & $5,48 \mathrm{a}$ \\
\hline CMF235 & $4,62 d$ & $0,54 \mathrm{~d}$ & $14,05 \mathrm{e}$ & $9,09 \mathrm{f}$ & $1,55 \mathrm{c}$ & $0,11 \mathrm{~b}$ & $4,88 \mathrm{f}$ & $0,83 \mathrm{~b}$ & $0,08 \mathrm{~b}$ & $9,73 b$ & $131,18 \mathrm{~b}$ & $5,39 \mathrm{~b}$ \\
\hline Golden & $3,89 \mathrm{e}$ & $0,39 d$ & $12,53 \mathrm{f}$ & $7,86 \mathrm{~g}$ & $1,60 \mathrm{c}$ & $0,15 \mathrm{a}$ & $4,55 \mathrm{~g}$ & $1,70 \mathrm{a}$ & $0,07 \mathrm{c}$ & $7,95 \mathrm{c}$ & $117,88 \mathrm{~b}$ & $5,59 \mathrm{a}$ \\
\hline CMF-L12-08 & $5,89 \mathrm{c}$ & $0,99 \mathrm{c}$ & $21,30 \mathrm{~b}$ & $9,63 \mathrm{e}$ & $2,21 \mathrm{a}$ & $0,12 b$ & $5,05 \mathrm{f}$ & $1,79 \mathrm{a}$ & $0,07 \mathrm{c}$ & $10,73 a$ & $163,99 a$ & $5,56 \mathrm{a}$ \\
\hline CMF-L30-08 & $6,11 \mathrm{c}$ & $0,69 \mathrm{c}$ & $18,93 \mathrm{c}$ & $8,98 \mathrm{f}$ & $2,12 \mathrm{a}$ & $0,11 b$ & $4,67 \mathrm{~g}$ & $2,24 \mathrm{a}$ & $0,07 \mathrm{c}$ & $7,25 \mathrm{c}$ & $104,43 \mathrm{c}$ & $5,48 \mathrm{a}$ \\
\hline CMF-L48-08 & $4,47 \mathrm{e}$ & $0,78 \mathrm{c}$ & $19,29 \mathrm{c}$ & $9,11 \mathrm{f}$ & $2,15 \mathrm{a}$ & $0,11 b$ & $4,53 \mathrm{~g}$ & $1,69 \mathrm{a}$ & $0,09 b$ & $9,98 \mathrm{~b}$ & $118,18 \mathrm{~b}$ & $5,35 \mathrm{~b}$ \\
\hline CMF-L62-08 & $5,38 \mathrm{~d}$ & $0,75 \mathrm{c}$ & $16,20 \mathrm{e}$ & $8,95 \mathrm{f}$ & $1,72 \mathrm{c}$ & $0,11 b$ & $5,43 \mathrm{f}$ & $1,10 \mathrm{~b}$ & $0,08 \mathrm{~b}$ & $10,08 \mathrm{~b}$ & $133,05 \mathrm{~b}$ & $5,45 \mathrm{a}$ \\
\hline CMF-L75-08 & $4,11 \mathrm{e}$ & $0,89 \mathrm{c}$ & $20,04 \mathrm{c}$ & $10,00 \mathrm{e}$ & $2,00 \mathrm{a}$ & $0,12 b$ & $4,68 \mathrm{~g}$ & $1,40 \mathrm{~b}$ & $0,05 \mathrm{c}$ & $8,78 \mathrm{c}$ & $167,64 a$ & $5,53 \mathrm{a}$ \\
\hline CMF-L88-08 & $6,13 \mathrm{c}$ & $0,81 \mathrm{c}$ & $19,84 \mathrm{c}$ & $9,61 \mathrm{e}$ & $2,07 \mathrm{a}$ & $0,11 b$ & $4,49 \mathrm{~g}$ & $0,90 \mathrm{~b}$ & $0,06 \mathrm{c}$ & $11,18 \mathrm{a}$ & $187,35 \mathrm{a}$ & $5,22 \mathrm{~b}$ \\
\hline CMF-L90-08 & $5,94 \mathrm{c}$ & $1,00 \mathrm{c}$ & $20,73 b$ & $10,31 \mathrm{e}$ & $2,04 \mathrm{a}$ & $0,11 b$ & $4,70 \mathrm{~g}$ & $1,36 \mathrm{~b}$ & $0,08 \mathrm{~b}$ & $9,93 b$ & $124,54 \mathrm{~b}$ & $5,38 \mathrm{~b}$ \\
\hline Sunrise & $4,24 \mathrm{e}$ & $0,43 d$ & $13,18 \mathrm{f}$ & $8,48 \mathrm{f}$ & $1,58 \mathrm{c}$ & $0,12 \mathrm{~b}$ & $5,09 \mathrm{f}$ & $1,23 \mathrm{~b}$ & $0,07 \mathrm{c}$ & $10,60 \mathrm{a}$ & $156,08 \mathrm{a}$ & $5,50 \mathrm{a}$ \\
\hline
\end{tabular}

${ }^{(1)}$ CPE-FR, comprimento do pedúnculo do fruto (cm); PFR, massa do fruto (g); CFR, comprimento do fruto (cm); LFR, largura do fruto (cm); RCFR/LFR, razão entre comprimento e largura de fruto; ECAS-FR, espessura da casca do fruto ( $\mathrm{cm}$ ); CCAV-FR, comprimento da cavidade central do fruto ( $\mathrm{cm}$ ); FIR-FR, firmeza do fruto (libras); ATT, acidez total titulável (g de ácido cítrico por 100 g); SST, sólidos solúveis totais ( ${ }^{\circ}$ Brix); Ratio, razão entre SST e ATT. Médias seguidas de letras iguais não diferem pelo teste Scott-Knott, a 5\% de probabilidade. 
$(0,13$ a $0,15 \mathrm{~cm})$, e o restante dos genótipos foram classificados conjuntamente, sem diferença significativa entre eles (variação de 0,10 a $0,12 \mathrm{~cm}$ ).

Os valores para cavidade interna dos frutos variaram de 3,69 a 10,64 cm (Tabela 4). Os genótipos com menor diâmetro da cavidade interna foram CMF230, CMF018, L88-08, CMF234, L48-08, Golden, L30-08, L75-08, L90-08 e CMF087, com variação entre 3,69 e $7,71 \mathrm{~cm}$. Este descritor está relacionado à qualidade dos frutos, pois aqueles com menor diâmetro da cavidade interna, geralmente, apresentam maior quantidade de polpa. Oliveira et al. (2010) observaram correlação significativa e positiva, embora de baixa magnitude $(r=0,42 *)$, entre espessura da polpa e firmeza de fruto.

Foram observados dois agrupamentos quanto à firmeza de frutos, um com valores entre 1,69 e 2,24 libras, para os genótipos L48-08, Golden, CMF041, L12-08, CMF008, CMF020, CMF154, CMF234, CMF024 e L30-08, e outro com valores inferiores a 1,5 libras, para os demais genótipos. A firmeza é um dos principais atributos na avaliação da qualidade do mamão, pois frutos com pouca firmeza apresentam menor resistência ao transporte, armazenamento e manuseio (Fagundes \& Yamanishi, 2001). Alguns estudos indicam firmeza de frutos de 1,23 a 2,29 libras, em variedades do grupo Solo, e de 3,95 a 6,31 libras, em variedades do grupo Formosa, quando avaliados em diferentes épocas de colheita (Fioravanço et al., 1994; Fagundes \& Yamanishi, 2001). No presente trabalho, os frutos foram avaliados em estádios mais avançados de maturação (nota 5) e, portanto, é esperado que as estimativas de firmeza de frutos estejam subestimadas, se comparadas às observadas nos trabalhos citados.

Os valores de acidez total titulável foram muito parecidos, apesar das diferenças significativas entre as médias dos genótipos, com variação de 0,05 a $0,14 \%$. Estes valores foram maiores do que a variação de 0,012 a $0,034 \%$, observada por Alonso et al. (2008), em experimento conduzido em Cuba com o objetivo de avaliar o comportamento produtivo das variedades Golden, BH-65, Baixinho de Santa Amália e Sunset. A diferença no ponto de colheita dos frutos também pode ter influenciado estes valores.

Com relação ao teor de sólidos solúveis, os genótipos CMF088, CMF041, CMF234, CMF087, Sunrise, L12-08, L88-08, CMF092 e CMF065 apresentaram os maiores valores, com variações de 10,35 a $11,53{ }^{\circ}$ Brix (Tabela 4). Onze genótipos não diferiram significativamente quanto a este caráter, com valores entre 9,38 e $10,08^{\circ} \mathrm{Brix}$, enquanto outros sete genótipos - L30-08, CMF024, Golden, CMF020, CMF008, L75-08 e CMF154 - apresentaram as menores médias (7,25 a 9,08 ${ }^{\circ}$ Brix). Os genótipos com maiores valores de SST também apresentaram maiores valores de Ratio. Em germoplasma, esta amplitude é ainda maior, com variação de 4,6 a 13,3 ${ }^{\circ}$ Brix (Ocampo et al., 2006).

De maneira geral, os valores de SST foram baixos para todos os genótipos, embora com amplitude dentro da observada na literatura, a exemplo dos valores entre: 7,85 e $12,65^{\circ} \mathrm{Brix}$, observados na análise de híbridos de mamoeiro por Marin et al. (2006); 5,00 e 16,20 ${ }^{\circ}$ Brix, na análise de germoplasma por Oliveira et al. (2010); 10,24 a $12,27^{\circ}$ Brix, na análise de populações segregantes por Silva et al. (2008a).

Apesar de muitos dos descritores avaliados não apresentarem importância direta para os programas de melhoramento, suas variações podem ser úteis na seleção indireta de genótipos com características desejáveis. Oliveira et al. (2010) constataram que as características largura de folha, altura de planta, número de flores por inflorescência e acidez total titulável apresentam efeitos diretos positivos e significativos para a seleção de plantas com maior número de frutos.

Apesar da significância e da separação das médias em dois grupos, não foram observadas grandes variações nos valores de $\mathrm{pH}$ da polpa dos frutos. As estimativas estiveram entre 5,22 e 5,64, sem relação direta com a ATT (Tabela 4).

Com relação à massa das sementes, a amplitude dos genótipos foi de 20,52 a 125,40 g de sementes frescas e de 5,46 a 24,65 g de sementes secas por fruto. A relação entre massa de matéria fresca e seca de sementes é linear, e os genótipos CMF012, CMF024 e CMF021 foram os que apresentaram maiores valores quanto a estes descritores. Embora não figurem entre os genótipos com maior quantidade de sementes, CMF018, CMF123, L12-08, CMF008 e Calimosa apresentaram as maiores dimensões de sementes, com comprimento entre $0,62 \mathrm{e}$ $0,65 \mathrm{~cm}$, e largura de 0,44 a $0,51 \mathrm{~cm}$ (Tabela 5). Em razão da relação entre comprimento e largura de sementes, observa-se que os genótipos CMF008, L30-08, L90-08 e CMF065 tendem a apresentar sementes elípticas, enquanto os genótipos CMF088, CMF154, CMF087, CMF021, CMF092, CMF024, CMF041 e CMF012 tendem a apresentar sementes alongadas. 


\section{Conclusões}

1. Há ampla variação e amplitude dos descritores relacionados a planta, folhas, frutos, flores e sementes do mamoeiro.

2. Há diferenças agronômicas suficientes para uso dos genótipos "per se" ou como parentais em programas de melhoramento, em razão de agregarem variação genética, qualidade de frutos e tipo agronômico.

3. As estimativas dos parâmetros genéticos mostram alta herdabilidade quanto à maioria dos descritores avaliados, o que indica seu potencial de uso na discriminação de genótipos, quando do registro e proteção de cultivares.

\section{Referências}

ALONSO, M.; TORNET, Y.; ARANGUREN, M.; RAMOS, R.; RODRÍGUEZ, K.; PASTOR, M.C.R. Caracterización de los frutos de cuatro cultivares de papaya del grupo Solo, introducidos en Cuba. Agronomía Costarricense, v.32, p.169-175, 2008.

BADILLO, V.M. Carica L. vs. Vasconcella St. Hil. (Caricaceae): con la rehabilitación de este último. Ernstia, v.10, p.74-79, 2000.

CARDOSO, D.L.; SILVA, R.F. da; PEREIRA, M.G.; VIANA, A.P.; ARAÚJO, E.F. Diversidade genética e parâmetros genéticos relacionados à qualidade fisiológica de sementes em germoplasma de mamoeiro. Revista Ceres, v.56, p.572-579, 2009.

CRUZ, C.D. Programa Genes: estatística experimental e matrizes. Viçosa: UFV, 2006. v.1, 285p.

CRUZ, C.D.; CARNEIRO, P.C.S. Modelos biométricos aplicados ao melhoramento genético. Viçosa: UFV, 2003. v.2, 585p.

DANTAS, J.L.L.; LIMA, J.F. de. Seleção e recomendação de variedades de mamoeiro - avaliação de linhagens e híbridos. Revista Brasileira de Fruticultura, v.23, p.617-621, 2001.

FAGUNDES, G.R.; YAMANISHI, O.K. Características físicas e químicas de frutos de mamoeiro do grupo Solo comercializados em quatro estabelecimentos de Brasília-DF. Revista Brasileira de Fruticultura, v.23, p.541-545, 2001.

FIORAVANÇO, J.C.; PAIVA, M.C.; CARVALHO, R.I.N. de; MANICA, I. Características do mamão Formosa comercializado em Porto Alegre de outubro/91 a junho/92. Ciência Rural, v.24, p.519-522, 1994.

FOLTRAN, D.E.; GONÇALVES, P. de S.; SABINO, J.C.; IGUE, T.; VILELA, R.C.F. Estimativas de parâmetros genéticos e fenotípicos em mamão. Bragantia, v.52, p.7-15, 1993.

FOOD AND AGRICULTURE ORGANIZATION OF THE UNITED NATIONS. FAO home. Available at: $<$ http//www.fao. org>. Accessed on: 10 Apr. 2011.
FRAIFE FILHO, G. de A.; DANTAS, J.L.L.; LEITE, J.B.V.; OLIVEIRA, J.R.P. Avaliação de variedades de mamoeiro no extremo sul da Bahia. Magistra, v.13, p.37-41, 2001.

GOMES, F.P. Curso de estatística experimental. 11.ed. São Paulo: Nobel, 1985. 467p.

INSTITUTO BRASILEIRO DE GEOGRAFIA E ESTATÍSTICA. Sistema IBGE de Recuperação Automática - SIDRA. Disponível em: <http://www.ibge.gov.br>. Acesso em: 23 abr. 2011.

INTERNATIONAL PLANT GENETIC RESOURCES INSTITUTE. Descriptor list for papaya. Rome: IPGRI, 1988. $34 \mathrm{p}$.

MARIN, S.L.D.; PEREIRA, M.G.; AMARAL JUNIOR, A.T. do; MARTELLETO, L.A.P.; IDE, C.D. Heterosis in papaya hybrids from partial diallel of Solo and Formosa parents. Crop Breeding and Applied Biotechnology, v.6, p.24-29, 2006.

NAKASONE, H.Y.; LAMOUREUX, C. Transitional forms of hermaphroditic papaya flowers leading to complete maleness. Journal of the American Society for Horticultural Science, v.107, p.589-592, 1982.

NISHIJIMA, W. Papaya. In: PLOETZ, R.C.; ZENTMYER, G.A.; NISHIJIMA, W.T.; ROHRBACH, K.G.; OHR, H.D. (Ed.). Compendium of tropical fruit disease. St. Paul: American Phytopathological Society, 1994. p.56-70.

OCAMPO, J.; D'EECKENBRUGGEB, G.C.; BRUYÉRE, S.; BELLAIRE, L. de L. de; OLLITRAULT, P. Organization of morphological and genetic diversity of Caribbean and Venezuelan papaya germplasm. Fruits, v.61, p.25-37, 2006.

OLIVEIRA, E.J. de; LIMA, D.S. de; LUCENA, R.S.; MOTTA, T.B.N.; DANTAS, J.L.L. Correlações genéticas e análise de trilha para número de frutos comerciais por planta em mamoeiro. Pesquisa Agropecuária Brasileira, v.45, p.855-862, 2010.

SILVA, F.F. da; PEREIRA, M.G.; RAMOS, H.C.C.; DAMASCENO JUNIOR, P.C.; PEREIRA, T.N.S.; GABRIEL, A.P.C.; VIANA, A.P.; DAHER, R.F.; FERREGUETTI, G.A. Estimation of genetic parameters related to morpho-agronomic and fruit quality traits of papaya. Crop Breeding and Applied Biotechnology, v.8, p.65-73, 2008a.

SILVA, F.F. da; PEREIRA, M.G.; RAMOS, H.C.C.; DAMASCENO JUNIOR, P.C.; PEREIRA, T.N.S.; GABRIEL, A.P.C.; VIANA, A.P.; FERREGUETTI, G.A. Selection and estimation of the genetic gain in segregating generations of papaya (Carica papaya L.). Crop Breeding and Applied Biotechnology, v.8, p.1-8, $2008 \mathrm{~b}$.

SILVA, F.F. da; PEREIRA, M.G.; RAMOS, H.C.C.; DAMASCENO JUNIOR, P.C.; PEREIRA, T.N.S.; IDE, C.D. Genotypic correlations of morpho-agronomic traits in papaya and implications for genetic breeding. Crop Breeding and Applied Biotechnology, v.7, p.345-352, 2007.

SINGH, K.; KUMAR, A. Genetic variability and correlation studies in papaya under Bihar conditions. Acta Horticulturae, v.851, p.145-150, 2010.

$\overline{\text { Recebido em } 21 \text { de maio de } 2011 \text { e aprovado em } 29 \text { de setembro de } 2011}$ 